\title{
BMJ Open Association between objectively measured body composition, sleep parameters and physical activity in preschool children: a cross-sectional study
}

Justyna Wyszyńska (10 , ${ }^{1}$ Piotr Matłosz (D) , ${ }^{2}$ Muhammad Asif, ${ }^{3}$ Agnieszka Szybisty, ${ }^{2}$ Paweł Lenik, ${ }^{2}$ Katarzyna Deren, ${ }^{1}$ Artur Mazur, ${ }^{4}$ Jarosław Herbert ${ }^{(D)}{ }^{2}$

To cite: Wyszyńska J, Matłosz P, Asif M, et al. Association between objectively measured body composition, sleep parameters and physical activity in preschool children: a crosssectional study. BMJ Open 2021;11:e042669. doi:10.1136/ bmjopen-2020-042669

- Prepublication history for this paper is available online. To view these files, please visit the journal online (http://dx.doi org/10.1136/bmjopen-2020042669).

Received 10 July 2020 Revised 12 December 2020 Accepted 10 January 2021

\section{Check for updates}

\section{Author(s) (or their} employer(s)) 2021. Re-use permitted under CC BY-NC. No commercial re-use. See rights and permissions. Published by BMJ.

${ }^{1}$ Institute of Health Sciences, Medical College, University of Rzeszów, Rzeszow, Poland ${ }^{2}$ Institute of Physical Culture Sciences, Medical College, University of Rzeszów, Rzeszow, Poland

${ }^{3}$ Department of Statistics, Govt. Degree College, Qadir Pur Raan, Multan, Pakistan

${ }^{4}$ Institute of Medical Sciences, Medical College, University of Rzeszów, Rzeszow, Poland

Correspondence to Dr Justyna Wyszyńska; justyna.wyszynska@onet.pl

\section{ABSTRACT}

Objective Associations between self-reported sleep duration and obesity indices in children are well recognised; however, there are no studies on associations between objectively measured other sleep parameters and physical activity with body composition in preschoolers. Therefore, the aim of this study was to determine the associations between sleep parameters and moderate-tovigorous physical activity (MVPA) with body composition indices in preschoolers using objective measures.

Design A cross-sectional study.

Participants The study group consisted of 676 children aged 5-6 years, who were enrolled in kindergartens in the 2017/2018 school year.

Outcome measures Sleep parameters and MVPA were measured using accelerometers for 7 days. Bioelectrical impedance analysis was used to estimate body composition.

Results Sleep duration and sleep efficiency were inversely associated with body fat percentage (BFP) ( $\beta=-0.013$ and $\beta$ from -0.311 to -0.359 , respectively) and body mass index (BMI) ( $\beta$ from -0.005 to -0.006 and from -0.105 to -0.121 , respectively), and directly associated with fat-free mass (FFM) ( $\beta$ from 0.010 to 0.011 and from 0.245 to 0.271 , respectively) and muscle mass ( $\beta$ from 0.012 to 0.012 and from 0.277 to 0.307 , respectively) in unadjusted and adjusted models. BFP was inversely associated with MVPA and positively associated with number of awakenings and sleep periods. Number of sleep periods was inversely associated with FFM, and positively with $\mathrm{BMI}$ and muscle mass. Correlation matrix indicated significant correlation between BFP, FFM and muscle mass with sleep duration, sleep efficiency, number of sleep periods and MVPA.

Conclusions Periodic assessment of sleep parameters and MVPA in relation to body composition in preschool children may be considered, especially in those who are at risk for obesity.

\section{INTRODUCTION}

Sleep is a physiological process that plays a vital role in brain function and systemic physiology across many body systems. Sufficient sleep quality is important for mental, emotional and physical health. ${ }^{1}$ Sleep
Strengths and limitations of this study

- In this study, we used a device-based measure of sleep, physical activity (actigraphy) and body composition (bioelectrical impedance analysis method).

- The $5 \mathrm{~s}$ epoch used in this study appears to capture a greater amount of data in preschool children.

- Energy intake was not adjusted for in analyses.

- Hip worn accelerometers for assessment of sleep quality metrics can be recognised as a limitation of the study.

disorders are common in childhood and adolescence. The overall prevalence of chronic sleep disruption among a random sample of 20505 children aged 5-12 years was $9.8 \%$ (boys, $10.0 \%$; girls, $8.9 \%$ ). ${ }^{2}$ Longterm consequences of sleep disruption in healthy individuals include hypertension, dyslipidaemia, cardiovascular disease, weightrelated issues, type 2 diabetes mellitus, gastrointestinal disorders and depression. ${ }^{3}$

Recent studies have reported that short sleep duration is a predictor of obesity in children and adolescents. A meta-analysis of 11 longitudinal studies, comprising almost 25000 children and adolescents, revealed that subjects sleeping for short duration had twice the risk of being overweight/obese, compared with subjects sleeping for long duration. ${ }^{4} \mathrm{~A}$ more recent systematic review and meta-analysis of prospective studies also indicated that children and adolescents with short sleep duration have increased odds for obesity. ${ }^{5}$ Nevertheless, some studies have reported that either short as well as long sleep duration are associated with increased prevalence of obesity. ${ }^{6}$

Sleep duration is not the only parameter that can affect weight-related issues. Other sleep parameters such as number of sleep periods, sleep efficiency, number of 
awakenings or length of awakenings can also affect body composition. It was found that longer sleep duration and higher sleep efficiency were associated with lower body mass index (BMI) among adults from the general population. ${ }^{7}$ Conversely, greater BMI and body fat percentage (BFP) were associated with lower sleep efficiency and longer wake after sleep onset (WASO). Elevated BMI or BFP were also observed for later wake times, shorter sleep duration and longer sleep latency. ${ }^{8}$ The relationship between sleep parameters with fat-free mass (FFM) and muscle mass remains inconsistent. For example, the Family Lifestyle, Activity, Movement and Eating (FLAME) study of children aged 3-7 years, demonstrated a significant association between sleep duration and fat mass but the relationship with FFM was non-significant. ${ }^{9}$ However, the opposite results were obtained in Korean adolescent boys. Self-reported sleep duration was inversely associated with BMI, BFP and positively with skeletal muscle index. ${ }^{10}$ Similarly, shorter sleep duration was associated with higher BMI and FFM index among children aged from 3 to 4 years. ${ }^{11}$ A recent experimental study in young men demonstrated that acute sleep loss contributed to decrease in levels of structural proteins in skeletal muscle and increase in levels of proteins linked to adipogenesis in adipose tissue. ${ }^{12}$

Current evidence has so far often been limited to studies using self-reported sleep analysis that include mainly sleep duration and BMI. To the best of our knowledge, there are no reports on the analysis of device measured sleep parameters and moderate-to-vigorous physical activity (MVPA) with body composition among large sample of preschool children. Therefore, we explored the association between sleep metrics, MVPA and body composition. Based on previous studies we hypothesised that: (1) BFP is inversely associated with sleep duration, sleep efficiency, MVPA and positively associated with WASO, number of awakening and number of sleep periods; (2) FFM is positively associated with sleep duration, sleep efficiency, MVPA and inversely associated with WASO, number of awakening, number of sleep periods; (3) muscle mass is positively associated with sleep duration, sleep efficiency, MVPA and inversely associated with WASO, number of awakening, number of sleep periods.

\section{METHODS}

\section{Study design and study sample}

A cross-sectional study was conducted at 22 kindergartens in Rzeszów, Poland. The study included healthy preschoolers aged 5-6 years. Participation in the study was voluntary and anonymous. Anthropometric measurements were taken between 08:00 and 10:00 by experienced researchers. Data were collected in the 2017/2018 school year, when preschoolers were attending kindergarten and excluded major holidays.

An invitation to participate in the study was sent to all parents of children aged between 5 and 6 years attending kindergartens. The consent of 707 parents was obtained for child participation in the measurements for the purpose of this study. Of those respondents, 31 were excluded from the study for the following reasons: a functional state that does not allow for self-maintenance of a standing position $(\mathrm{n}=1)$, strong anxiety of examination $(\mathrm{n}=3)$, taking medication affecting body composition $(n=3)$, a failure to return or complete the survey $(n=18)$ or lack of valid accelerometer data $(n=6)$. Ultimately, the study group consisted of 676 students (51\% girls). The margin of error for study group consisted of 676 individuals is maximum $4 \%$.

\section{Patient and public involvement}

Patients or the public were not involved in the design, or conduct, or reporting, or dissemination plans of our research.

\section{Anthropometric measurements}

Anthropometric measurements (body mass, body height) were performed under standard conditions. Body height was measured in an upright position, barefoot, to the nearest $0.1 \mathrm{~cm}$ using a portable stadiometer (Tanita HR-200). Body mass was assessed with an accuracy of $0.01 \mathrm{~kg}$ using a body composition analyser (BC-420 MA, Tanita). BMI was calculated as body mass $(\mathrm{kg})$ divided by height $(\mathrm{m})$ squared. Based on BMI values, the BMI percentile of individual participants were calculated. Polish BMI percentile charts specific for age, gender and body height were used..$^{13}$ Based on the BMI percentile values, underweight, healthy weight, overweight and obesity were determined. ${ }^{14}$

\section{Physical activity and sleep}

Actigraphic sleep parameters and MVPA data were collected using the tri-axial accelerometer (GT3X-BT Monitor; ActiGraph, Pensacola, Florida, USA) and were analysed using the ActiLife 6.13 data analysis software. Actigraphy is a valid, effective and cost-efficient alternative to polysomnography ${ }^{15}$ and widely used to objectively measure physical activity level in preschool children. ${ }^{16}$ The device was attached at the participant's right hip Participants were instructed to wear the monitor for 24 hours daily for seven consecutive days and nights during all activities, except for water-related activities. Parents or caregivers also received detailed instructions on the use of the activity monitor by their children.

As the accelerometer was worn for 24 hours per day, it was necessary to identify nocturnal sleep episode time distinct, and this was done using a published automated algorithm. ${ }^{17}$ Actigraphy data were collected at a sampling rate of $30 \mathrm{~Hz}$ and the 'Sadeh' sleep algorithm was used. ${ }^{18}$ In the current study, the following five sleep outcomes were examined: sleep duration (total sleep time), sleep efficiency, WASO, number of sleep periods and number of awakenings. Sleep duration refers to the number of 60 s epochs in a sleep episode scored as 'sleep', excluding any time scored as 'wake'. Sleep efficiency is percentage of total time in bed actually spent in sleep. WASO is the 
duration (in minutes) of wake time in a sleep episode after sleep has been initiated. Sleep periods is defined as the total number of sleep periods that were detected in the data set, and number of awakenings is the frequency of awakenings during a sleep episode. ${ }^{19}$

After exclusion of the nocturnal sleep episode time, non-wear time was determined as $60 \mathrm{~min}$ of consecutive zeros allowing for $2 \mathrm{~min}$ of non-zero interruptions. ${ }^{20}$ Once nocturnal sleep episode time and non-wear time were computed, waking wear time and the physical activity levels were calculated and identified using the $5 \mathrm{~s}$ epoch data. The findings suggested that using $5 \mathrm{~s}$ epochs are better adapted to the preschool children physical activity patterns..$^{21}$ A waking wear time of $\geq 500 \mathrm{~min} /$ day was used as the criterion for a valid day, and $\geq 4$ days were used as the criteria for a valid 7-day period of accumulated data (including $\geq 3$ valid weekdays and $\geq 1$ valid weekend day). ${ }^{22}$ For each participant, the mean MVPA (min/day) and the mean daily step count were calculated. The cut-off points from Evenson et al were selected to determine the time spent on MAPA. MVPA time was calculated as the mean daily minutes $\geq 2296$ counts $/ \mathrm{min}$ from all valid days. ${ }^{23}$

The majority of children $(\mathrm{n}=741,77.8 \%)$ had complete 7-day actigraphic sleep data; 170 (17.8\%) and 42 (4.4\%) children had 6-day and 5-day actigraphic data, respectively. Average monitoring included 6.7 days of recordings.

\section{Body composition}

Foot-to-foot bioelectrical impedance analysis (BIA) was used to estimate body composition with a body composition analyzer (BC-420, Tanita). The foot-to-foot method of BIA is a reliable and accurate tool for the measurement of body composition in the paediatric population. ${ }^{24}$ Results showed strong significant correlations between dual-energy X-ray absorptiometry (DEXA) and BIA for fat mass, BFP and FFM..$^{25}$

BIA was performed in the early morning after overnight fasting for at least 8 hours since food or beverage consumption may decrease impedance by $4-15$ over a 2-4hours period after meals, representing an error smaller than $3 \% .{ }^{26}$ Body composition was differentiated into BFP, FFM and muscle mass percentage.

\section{Statistical analysis}

Statistical analysis was performed using the SPSS V.21 software. Descriptive statistics are presented as mean $( \pm \mathrm{SD})$ and $n(\%)$. All variables were checked for normality before the analysis using Kolmogorov-Smirnoff test. Group differences between means were analysed with Mann-Whitney U test and Pearson's $\chi^{2}$ test. Simple and multiple linear regression models were used to examine the independent associations of sleep parameters and MVPA with body composition indices (ie, BFP\%, FFM\%, muscle mass and BMI). The multicollinearity between independent variables was determined using variance inflation factor. The variance inflation factors between variables were $<5$, suggesting that multicollinearity was not a problem in the models. The primary model (model
1) was unadjusted, each association between variables was constructed separately by simple linear regression. Model 2 was adjusted for age and gender. Model 3 and model 4 were consisted of model 2 plus MVPA and sleep duration, respectively. A correlation matrix, using Spearman's correlation coefficients ( $r$ ), was determined to examine the associations between body composition indices with the sleep parameters and MVPA. The significance level was set at $\mathrm{p} \leq 0.05$.

\section{RESULTS}

The final sample consisted of 676 preschoolers aged 5-6 years (mean age 5.55years). Mean height was $117.21 \pm 6.06 \mathrm{~cm}$ and mean weight $21.67 \pm 3.89 \mathrm{~kg}$. There were no significant differences in age, body composition and sleep parameters between boys and girls. Mean MVPA were significantly lower in girls than in boys $(\mathrm{p}<0.001)$ (table 1).

Table 2 shows the independent associations of sleep parameters and MVPA with body composition. Sleep duration, sleep efficiency and MVPA were inversely associated with BFP in unadjusted and adjusted models. Number of sleep periods and number of awakenings were directly associated with BFP in unadjusted models and after adjusting for age and gender (model 2). Associations between number of sleep periods and BFP remained significant after further adjustment for MVPA (model 3). FFM was positively associated with sleep duration and sleep efficiency, and negatively associated with number of sleep periods in unadjusted and adjusted models. Number of awakenings was negatively associated with FFM after adjusting for age and gender (model 2). Sleep duration and sleep efficiency were directly associated with muscle mass in all models. Positive significant association was also observed between MVPA and muscle mass after adjusting for age, gender and sleep duration (model 4). BMI were inversely associated with sleep duration and sleep efficiency, and directly associated with number of sleep periods in unadjusted and adjusted models.

Correlation matrix for sleep parameters, MVPA and body composition indicated significant correlation between BFP and sleep duration $(\mathrm{r}=-0.110)$, sleep efficiency $(r=-0.090)$, number of sleep periods $(r=0.116)$ and MVPA $(r=-0.095)(\mathrm{p} \leq 0.01)$. Positive correlation was also found between FFM and muscle mass with sleep duration, sleep efficiency, MVPA and inverse correlation with number of sleep periods (table 3).

\section{DISCUSSION}

Most available studies examined the relationship between self-reported sleep duration and obesity indicators such as BMI, waist circumference or skinfold thickness. More research is needed to fully understand how other devicebased sleep measures and physical activity are associated with body composition, as these may represent different aspects of sleep compared with sleep duration. Therefore, 
Table 1 Characteristics of the study population

\begin{tabular}{|c|c|c|c|c|}
\hline Variable & All subjects $(n=676)$ & Boys $(n=331)$ & Girls (n=345) & $P$ value \\
\hline Age (years) ${ }^{\star}$ & $5.55 \pm 0.50$ & $5.55 \pm 0.50$ & $5.56 \pm 0.50$ & 0.742 \\
\hline Height $(\mathrm{cm})^{\star}$ & $117.21 \pm 6.06$ & $117.90 \pm 6.13$ & $116.55 \pm 5.92$ & 0.006 \\
\hline BMI $\left(\mathrm{kg} / \mathrm{m}^{2}\right)^{\star}$ & $15.68 \pm 1.76$ & $15.78 \pm 1.81$ & $15.57 \pm 1.70$ & 0.147 \\
\hline \multicolumn{5}{|l|}{ Body mass category† } \\
\hline Normal & $540(79.9)$ & $274(82.8)$ & $266(77.1)$ & \\
\hline Overweight & $46(6.8)$ & $22(6.6)$ & $24(7.0)$ & \\
\hline Obesity & $30(4.4)$ & $16(4.8)$ & $14(4.1)$ & \\
\hline $\operatorname{BFP}(\%)^{*}$ & $20.36 \pm 4.73$ & $20.33 \pm 4.68$ & $20.38 \pm 4.79$ & 0.291 \\
\hline Muscle mass $(\%)^{*}$ & $76.65 \pm 4.66$ & $76.52 \pm 4.35$ & $76.77 \pm 4.94$ & 0.619 \\
\hline Sleep duration $(\min )^{\star}$ & $573.89 \pm 66.32$ & $570.11 \pm 62.47$ & $577.51 \pm 69.70$ & 0.077 \\
\hline Sleep efficiency $(\%)^{\star}$ & $97.32 \pm 1.50$ & $97.19 \pm 1.70$ & $97.44 \pm 1.25$ & 0.280 \\
\hline WASO (min)* & $14.29 \pm 6.32$ & $14.61 \pm 6.66$ & $13.97 \pm 5.97$ & 0.392 \\
\hline Number of awakenings* & $5.05 \pm 2.14$ & $5.21 \pm 2.22$ & $4.90 \pm 2.04$ & 0.122 \\
\hline Number of sleep periods* & $7.31 \pm 1.20$ & $7.29 \pm 1.20$ & $7.33 \pm 1.21$ & 0.916 \\
\hline
\end{tabular}

Significant associations are highlighted in bold.

${ }^{*}$ Mean \pm SD.

tn (\%).

BFP, body fat percentage; BMI, body mass index; FFM, fat-free mass; MVPA, moderate to vigorous physical activity; WASO, wake after sleep onset.

it was necessary to examine the associations between objectively measured sleep parameters and physical activity with body composition, including body fatness, as sleep disturbance and childhood obesity are serious health problems. ${ }^{27}$ The present results showed that in total sample of children aged from 5 to 6 years, lower sleep duration and sleep efficiency were significantly associated with higher BFP and BMI, and lower FFM and muscle mass. The number of sleep periods was directly associated with BFP and BMI, and inversely associated with FFM and muscle mass. Moreover, BFP were inversely associated with MVPA. There were no significant associations between the WASO and body composition indices.

In the general population, the literature is generally supportive of an association between the increased prevalence of sleep curtailment and the childhood obesity epidemic. ${ }^{2829}$ Cross-sectional $^{103031}$ as well as longitudinal studies ${ }^{32-34}$ have found that short sleep duration is associated with weight gain, higher BMI values and increased odds of being overweight or obese. This is supported also by meta-analyses ${ }^{28} 2935$; notwithstanding, some authors have not reached this conclusion. For example in a sample of children living in resource-limited settings in Peru, there was no association between short sleep duration and obesity. ${ }^{36}$ Moreover, it has been reported that association between sleep duration and obesity is not consistent in boys and girls, as some studies have reported this finding only in boys. ${ }^{37} 38$ Some studies discovered a relatively linear negative association between sleep duration and obesity ${ }^{39}$; however, others showed a U-shaped association that implicates both short and long sleep duration. ${ }^{40}$ Results of our study suggested that of sleep duration, sleep efficiency and number of sleep periods showed association with all body composition indices. Sleep duration showed the strongest associations with all body composition indicators, both in unadjusted and adjusted models. Several possible explanations have been presented to explain the biological plausibility of these associations. Sleep plays a critical role in metabolic and endocrine regulation and short sleep is associated with changes in metabolism. ${ }^{41}$ Insufficient sleep may lead to endocrine alternation, ${ }^{42}$ including decreased levels of leptin, glucose tolerance and insulin sensitivity and increased levels of ghrelin, hunger and appetite. ${ }^{43}$ Moreover, evidence suggests a link between short sleep and specific behaviour, such as low level of physical activity, high level of sedentary behaviour ${ }^{42}$ and food intake. ${ }^{44}$ This relationship is explained by energy balance hypothesis, which says that short sleep causes additional energy expenditures, thereby causing the body to automatically reserve energy through reducing activity and increasing food intake. ${ }^{45}$

The linear regression models of the present study suggested an association between FFM and muscle mass 
Table 2 Independent association of sleep parameters and MVPA with body composition indices

\begin{tabular}{|c|c|c|c|c|c|c|c|c|c|c|c|c|}
\hline \multirow[b]{2}{*}{ Variables } & \multicolumn{3}{|l|}{ BFP } & \multicolumn{3}{|l|}{ FFM } & \multicolumn{3}{|c|}{ Muscle mass } & \multicolumn{3}{|l|}{ BMI } \\
\hline & $\beta$ & $P$ value & $\mathbf{R}^{2}$ & $\beta$ & $P$ value & $\mathbf{R}^{2}$ & $\beta$ & $P$ value & $\mathbf{R}^{2}$ & $\beta$ & P value & $\mathbf{R}^{2}$ \\
\hline \multicolumn{13}{|c|}{ Sleep duration } \\
\hline Model 2 & -0.013 & $<0.001$ & 0.040 & 0.010 & $<0.001$ & 0.025 & 0.012 & $<0.001$ & 0.041 & -0.005 & $<0.001$ & 0.051 \\
\hline Model 3 & -0.013 & $<0.001$ & 0.053 & 0.011 & $<0.001$ & 0.029 & 0.012 & $<0.001$ & 0.047 & -0.006 & $<0.001$ & 0.055 \\
\hline Model 1 & -0.350 & 0.004 & 0.012 & 0.256 & 0.039 & 0.006 & 0.302 & 0.012 & 0.009 & -0.121 & 0.007 & 0.011 \\
\hline Model 2 & -0.359 & 0.003 & 0.020 & 0.271 & 0.029 & 0.012 & 0.307 & 0.010 & 0.020 & -0.114 & 0.012 & 0.017 \\
\hline Model 3 & -0.311 & 0.011 & 0.028 & 0.245 & 0.050 & 0.014 & 0.277 & 0.022 & 0.024 & -0.105 & 0.022 & 0.019 \\
\hline \multicolumn{13}{|l|}{ WASO } \\
\hline Model 1 & 0.040 & 0.160 & 0.003 & -0.044 & 0.129 & 0.003 & -0.017 & 0.543 & 0.001 & 0.010 & 0.349 & 0.001 \\
\hline \multicolumn{13}{|c|}{ Number of awakenings } \\
\hline Model 1 & 0.180 & 0.034 & 0.007 & -0.164 & 0.059 & 0.005 & -0.139 & 0.098 & 0.004 & 0.051 & 0.109 & 0.004 \\
\hline Model 2 & 0.185 & 0.030 & 0.014 & -0.171 & 0.049 & 0.010 & -0.138 & 0.099 & 0.015 & 0.047 & 0.139 & 0.010 \\
\hline Model 3 & 0.156 & 0.068 & 0.024 & -0.155 & 0.076 & 0.013 & -0.119 & 0.157 & 0.019 & 0.041 & 0.196 & 0.013 \\
\hline \multicolumn{13}{|c|}{ Number of sleep periods } \\
\hline Model 1 & 0.479 & 0.002 & 0.015 & -0.453 & 0.003 & 0.013 & -0.517 & 0.001 & 0.018 & 0.161 & 0.004 & 0.012 \\
\hline Model 2 & 0.463 & 0.002 & 0.021 & -0.440 & 0.004 & 0.017 & -0.501 & 0.001 & 0.027 & 0.167 & 0.003 & 0.020 \\
\hline Model 3 & 0.425 & 0.005 & 0.031 & -0.419 & 0.007 & 0.019 & -0.477 & 0.001 & 0.031 & 0.160 & 0.004 & 0.023 \\
\hline \multicolumn{13}{|l|}{ MVPA } \\
\hline
\end{tabular}

Model 1: unadjusted model; model 2: adjusted for age and gender; model 3: adjusted for age, gender and MVPA, model 4: adjusted for age, gender and sleep duration.

Significant associations are highlighted in bold.

BFP, body fat percentage; BMI, body mass index; FFM, fat-free mass; MVPA, moderate to vigorous physical activity; WASO, wake after sleep onset.

with sleep duration, sleep efficiency and number of sleep periods. Moreover, model adjusted for age, gender and sleep duration showed positive association between muscle mass and MVPA. However, no association was found between FFM and MVPA. Nonetheless, the correlation matrix additionally indicated an associations between all body composition indices and MVPA; however, these associations were very weak. The relationship of physical activity with muscle mass and FFM is poorly understood especially in paediatric population. Cross-sectional studies showed contradictory findings. Collings et al found that in preschoolers, physical activity variables including MVPA, were positively correlated with lean mass, but correlations were not statistically significant and there was no discernible pattern toward stronger correlations for higherintensity physical activity. ${ }^{46}$ However, Janz et al observed a positive association between MVPA and FFM in girls. ${ }^{47}$ The association between sleep indices with FFM and muscle mass remains inconsistent. Results of the present study support the previous findings obtained in Korean adolescent boys, whose self-reported sleep duration was positively with muscle mass. ${ }^{10}$ Among children aged from 3 to 4 years, shorter sleep duration was associated with higher FFM, ${ }^{11}$ while the FLAME study of children aged 3-7 years, demonstrated no relationship between sleep duration and FFM. ${ }^{9}$

Using a multivariate structural equation modelling, Bailey et al found that a model including sleep efficiency, sleep pattern inconsistency (latent variable consisting of the 7-day SD of bedtime, wake time, and sleep duration), and physical activity was the best for predicting BFP. ${ }^{1}$ Wirth et al determined relationships between poor sleep characteristics (ie, short sleep duration, low sleep efficiency, greater WASO) and BMI or body fat in adults. Individuals representing 'unhealthy' sleep had significantly elevated BMI and BFP. Greater BMI and BFP were 
Table 3 Correlation matrix for sleep parameters, MVPA and body composition indices

\begin{tabular}{|c|c|c|c|c|c|c|c|c|c|c|}
\hline Parameters & BFP & FFM & $\begin{array}{l}\text { Muscle } \\
\text { mass }\end{array}$ & BMI & $\begin{array}{l}\text { Sleep } \\
\text { duration }\end{array}$ & $\begin{array}{l}\text { Sleep } \\
\text { efficiency }\end{array}$ & WASO & $\begin{array}{l}\text { Awakenings } \\
\text { (n) }\end{array}$ & $\begin{array}{l}\text { Sleep } \\
\text { periods } \\
\text { (n) }\end{array}$ & MVPA \\
\hline BFP & 1 & $-0.853^{\star}$ & $-0.824^{*}$ & 0795* & $-0.110 \dagger$ & $-0.090 \dagger$ & $0.049^{N S}$ & $0.058^{\mathrm{NS}}$ & $0.116 \dagger$ & $-0.095 \dagger$ \\
\hline Muscle mass & $-0.824^{*}$ & $0.477^{\star}$ & 1 & $-0.654^{*}$ & $0.109 \dagger$ & $0.083 c$ & $-0.033^{\mathrm{NS}}$ & $-0.058^{\mathrm{NS}}$ & $-0.126^{\star}$ & $0.077 c$ \\
\hline BMI & $0.795^{\star}$ & $-0.702^{\star}$ & $-0.654^{\star}$ & 1 & $-0.156^{\star}$ & $-0.075 c$ & $0.029^{N S}$ & $0.036^{\mathrm{NS}}$ & $0.107 \dagger$ & $-0.015^{\mathrm{NS}}$ \\
\hline $\begin{array}{l}\text { Sleep } \\
\text { efficiency }\end{array}$ & $-0.090 \dagger$ & $0.079 c$ & $0.083 c$ & $-0.075 c$ & $0.303^{\star}$ & 1 & $-0.889^{\star}$ & $-0.769^{\star}$ & $-0.376^{\star}$ & $0.157^{\star}$ \\
\hline WASO & $0.049^{N S}$ & $-0.052^{\text {NS }}$ & $-0.033^{\mathrm{NS}}$ & $0.029^{\mathrm{NS}}$ & $-0.022^{\text {NS }}$ & $-0.889^{*}$ & 1 & $0.873^{*}$ & $0.141^{*}$ & $-0.142^{*}$ \\
\hline $\begin{array}{l}\text { Number of } \\
\text { awakenings }\end{array}$ & $0.058^{\mathrm{NS}}$ & $-0.044^{\mathrm{NS}}$ & $-0.058^{\mathrm{NS}}$ & $0.036^{\mathrm{NS}}$ & $-0.029^{N S}$ & $-0.769^{\star}$ & $0.873^{\star}$ & 1 & $0.106 \dagger$ & $-0.104 \dagger$ \\
\hline
\end{tabular}

${ }^{*} \mathrm{p} \leq 0.001$.

$\dagger \mathrm{p} \leq 0.01$.

$\neq \mathrm{p} \leq 0.05$.

BFP, body fat percentage; BMI, body mass index; FFM, fat-free mass; MVPA, moderate to vigorous physical activity; NS, not significant; WASO, wake after sleep onset.

associated with low sleep efficiency (BMI $=25.5$ vs $24.8 \mathrm{~kg}$ / $\mathrm{m}^{2} ; \mathrm{BFP}=27.7 \%$ vs $\left.26.5 \%\right)$ and high WASO (BMI $=25.6$ vs $25.0 \mathrm{~kg} / \mathrm{m}^{2} ; \mathrm{BFP}=28.0 \%$ vs $\left.26.7 \%\right)$. Elevated BMI or BFP also were observed for shorter sleep duration, later wake times and longer sleep latency. Gender modified the association between wake times and body composition. ${ }^{8}$ Partially different results were obtained in our study, which suggested that WASO was not significantly associated with any body composition indicators. Sleeprelated findings from our study are generally consistent with findings from previous studies using self-report or other actigraphy devices to characterise sleep, in that poor sleep quality have been associated with increased BMI or adipose tissue. ${ }^{48}$ The associations of sleep metrics with FFM and muscle mass, in the present study, are a novel finding. FFM and muscle mass were directly associated with sleep duration and sleep efficiency, and inversely associated with number of sleep periods, both in unadjusted and adjusted models. In addition, FFM was inversely associated with number of awakenings, and muscle mass was directly associated with MVPA after adjusting for age, gender and sleep duration.

The hypothesis of this study was partially confirmed because the correlation matrix showed that BFP was inversely associated with sleep duration, sleep efficiency, MVPA and positively associated with number of sleep periods. FFM and muscle mass were inversely associated with number of sleep periods, and positively associated with sleep duration, sleep efficiency and MVPA. No associations were found between body composition indices with WASO and number of awakenings. These findings support the hypothesis of a link between selected sleep measures, physical activity and BFP, FFM and muscle mass. The dependence of body composition on sleep behaviour needs to be investigated in experimental and longitudinal studies.

Findings from this study have important clinical and public health implications. The results indicated that children's sleep efficiency, number of awakening and number of sleep periods could be equally as influential on the sleep-adiposity association as the prolifically researched sleep duration. Childhood sleep duration and MVPA may be a modifiable risk factor in preventing alternation in body composition. Interventions to improve sleep quantity and quality could help to prevent and control excess adiposity and alternation in body composition among children. Our results suggested a relationship between sleep parameters, MVPA and body composition, therefore, poor sleep quality may be a risk factor, for example, fat tissue accumulation. On the other hand, increased body fat and the metabolic abnormalities may contribute to poor sleep quality. For instance, evidence suggested an association between altered glucose metabolism, fat tissue and poor sleep quality. ${ }^{50}$ Indeed, evidence have linked short sleep duration and poor sleep quality to excess weight risk; however, the underlying mechanism remains unclear. ${ }^{31}$ Further longitudinal studies are needed to provide insight into cause and effect. Understanding the complex relationships between sleep metrics and body composition indices could help to reduce the burden of obesity-related diseases by incorporating sleep components into weight loss interventions.

There are several strengths and limitations with the current study. Body composition was measured by using 
the BIA method that has demonstrated excellent testretest reliability, moderately strong absolute agreement with DEXA, and high specificity for overfat and obese classification. ${ }^{25}$ Another strength of this study was the use of hip worn accelerometers for assessment of MVPA and sleep quantity metrics. However, despite the fact that accelerometers can be used to assess physical activity and sleep, hip worn accelerometers for assessment of sleep quality metrics (sleep efficiency, WASO) can be recognised as a limitation of our study. According to a validation study with polysomnography in young children, a best site placement to estimate of all sleep parameters could not be determined, but overall the results suggested accelerometers at the hip may be superior for sleep timing and quantity metrics, whereas the wrist may be superior for sleep quality metrics. ${ }^{51}$ Another validation study among adult aged 50-75 years also indicated that for sleep duration, sleep efficiency, and WASO hip estimates differed significantly from polysomnography estimates. ${ }^{52}$ Another limitation of the study is cross-sectional design, thus the causal pathways underlying the observed relationships could hardly be detected. Energy intake was not adjusted for in analyses, which may compromise the results. Although measures of energy intake would have enriched our ability to interpret the results, lack of those measures and obtaining strong associations between sleep parameters and MVPA with body composition, does not negate the relevance of this study to public health. Short sleeping periods during the day (naps) and sedentary time was not analysed in present paper which could be recognised as another limitation of the study and recommendation for future research.

Future research should include a greater emphasis on longitudinal studies to examine how objectively measured sleep and fat content vary by age within an individual over time, taking into account possible confounding factors. Multiple longitudinal cohorts with different ages of participants would help to corroborate and extend our findings. Further research should also seek underlying mechanisms.

\section{CONCLUSIONS}

In conclusion, lower sleep duration and sleep efficiency were associated with higher BFP and BMI, and lower FFM and muscle mass. Higher number of sleep periods was associated with higher BFP and BMI, and lower FFM and muscle mass. Negative association was observed between BFP and MVPA. Periodic assessment of sleep parameters and physical activity in relation to body composition in preschool children may be considered, especially in those who are at risk for obesity.

Acknowledgements We are grateful to the participants and their families who volunteered their time to participate in this research.

Contributors Conceptualisation, JW. Data curation, JW and KD. Formal analysis, JW. Investigation, JW, PM, AS, PL, KD and JH. Methodology, JW and PM. Project administration, JW. Resources, JW and AM. Software, JW, MA and JH. Supervision,
JW. Writing—original draft, JW. Writing—review and editing, JW, PM, AS, MA and AM.

Funding The authors have not declared a specific grant for this research from any funding agency in the public, commercial or not-for-profit sectors.

Competing interests None declared.

Patient and public involvement Patients and/or the public were not involved in the design, or conduct, or reporting, or dissemination plans of this research.

Patient consent for publication Not required.

Ethics approval Written informed consent was obtained from parents or legal guardians, and child assent was also obtained before participation in the study. The study was approved by the Bioethics Committee at the Medical Department of the University of Rzeszów, decision no. 2018/01/05, and it was conducted in accordance with ethical standards laid down in an appropriate version of the Declaration of Helsinki.

Provenance and peer review Not commissioned; externally peer reviewed.

Data availability statement All data relevant to the study are included in the article or uploaded as supplementary information. All data relevant to the study are included in the article or uploaded as supplementary information.

Open access This is an open access article distributed in accordance with the Creative Commons Attribution Non Commercial (CC BY-NC 4.0) license, which permits others to distribute, remix, adapt, build upon this work non-commercially, and license their derivative works on different terms, provided the original work is properly cited, appropriate credit is given, any changes made indicated, and the use is non-commercial. See: http://creativecommons.org/licenses/by-nc/4.0/.

ORCID iDs

Justyna Wyszyńska http://orcid.org/0000-0002-5786-6214

Piotr Matłosz http://orcid.org/0000-0002-7388-5722

Jarosław Herbert http://orcid.org/0000-0003-0434-6263

\section{REFERENCES}

1 Bailey BW, Allen MD, LeCheminant JD, et al. Objectively measured sleep patterns in young adult women and the relationship to adiposity. Am J Health Promot 2014;29:46-54.

2 Li L, Ren J, Shi L, et al. Frequent nocturnal awakening in children: prevalence, risk factors, and associations with subjective sleep perception and daytime sleepiness. BMC Psychiatry 2014;14:204.

3 Medic G, Wille M, Hemels ME. Short- and long-term health consequences of sleep disruption. Nat Sci Sleep 2017;9:151-61.

4 Fatima Y, Doi SAR, Mamun AA. Longitudinal impact of sleep on overweight and obesity in children and adolescents: a systematic review and bias-adjusted meta-analysis. Obes Rev 2015;16:137-49.

5 Wu Y, Gong Q, Zou Z, et al. Short sleep duration and obesity among children: a systematic review and meta-analysis of prospective studies. Obes Res Clin Pract 2017;11:140-50.

6 Wang H, Hu R, Du H, et al. The relationship between sleep duration and obesity risk among school students: a cross-sectional study in Zhejiang, China. Nutr Metab 2018;15:48.

7 Koolhaas CM, Kocevska D, Te Lindert BHW, et al. Objectively measured sleep and body mass index: a prospective bidirectional study in middle-aged and older adults. Sleep Med 2019;57:43-50.

8 Wirth MD, Hébert JR, Hand GA, et al. Association between actigraphic sleep metrics and body composition. Ann Epidemiol 2015;25:773-8.

9 Carter PJ, Taylor BJ, Williams SM, et al. Longitudinal analysis of sleep in relation to $\mathrm{BMI}$ and body fat in children: the FLAME study. BMJ 2011;342:d2712.

$10 \mathrm{Nam}$ GE, Han K, Kim DH, et al. Sleep duration is associated with body fat and muscle mass and waist-to-height ratio beyond conventional obesity parameters in Korean adolescent boys. J Sleep Res 2017;26:444-52.

11 Baird J, Hill CM, Harvey NC, et al. Duration of sleep at 3 years of age is associated with fat and fat-free mass at 4 years of age: the Southampton Women's Survey. J Sleep Res 2016;25:412-8.

12 Cedernaes J, Schönke M, Westholm JO, et al. Acute sleep loss results in tissue-specific alterations in genome-wide DNA methylation state and metabolic fuel utilization in humans. Sci Adv 2018;4:eaar8590.

13 Kułaga Z, Różdżyńska-Świątkowska A, Grajda A. Percentile charts for growth and nutritional status assessment in Polish 
children and adolescents from birth to 18 year of age. Stand Med 2010;7:690-700.

14 Barlow SE, Expert Committee. Expert Committee recommendations regarding the prevention, assessment, and treatment of child and adolescent overweight and obesity: summary report. Pediatrics 2007;120:S164-92.

15 Sadeh A. The role and validity of actigraphy in sleep medicine: an update. Sleep Med Rev 2011;15:259-67.

16 Leeger-Aschmann CS, Schmutz EA, Zysset AE, et al. Accelerometerderived physical activity estimation in preschoolers - comparison of cut-point sets incorporating the vector magnitude vs the vertical axis. BMC Public Health 2019;19:513.

17 Tudor-Locke C, Barreira TV, Schuna JM, et al. Fully automated waistworn accelerometer algorithm for detecting children's sleep-period time separate from 24-h physical activity or sedentary behaviors. Appl Physiol Nutr Metab 2014;39:53-7.

18 Acebo C, Sadeh A, Seifer R, et al. Estimating sleep patterns with activity monitoring in children and adolescents: how many nights are necessary for reliable measures? Sleep 1999;22:95-103.

19 ActiGraph. ActiLife 6 user's manual, version 9.0.0, 2012. Available: https://s3.amazonaws.com/actigraphcorp.com/wp-content/uploads/ 2018/02/22094137/SFT12DOC13-ActiLife-6-Users-Manual-Rev-A110315.pdf [Accessed 6 Dec 2020].

20 Troiano RP, Berrigan D, Dodd KW, et al. Physical activity in the United States measured by accelerometer. Med Sci Sports Exerc 2008;40:181-8.

21 Vale S, Santos R, Silva P, et al. Preschool children physical activity measurement: importance of epoch length choice. Pediatr Exerc Sci 2009:21:413-20.

22 Hinkley T, O'Connell E, Okely AD, et al. Assessing volume of accelerometry data for reliability in preschool children. Med Sci Sports Exerc 2012;44:2436-41.

23 Evenson KR, Catellier DJ, Gill K, et al. Calibration of two objective measures of physical activity for children. J Sports Sci 2008;26:1557-65.

24 Tyrrell VJ, Richards G, Hofman P, et al. Foot-to-foot bioelectrical impedance analysis: a valuable tool for the measurement of body composition in children. Int J Obes Relat Metab Disord 2001;25:273-8.

25 Karelis AD, Chamberland G, Aubertin-Leheudre M, et al. Validation of a portable bioelectrical impedance analyzer for the assessment of body composition. Appl Physiol Nutr Metab 2013;38:27-32.

26 Kushner RF, Gudivaka R, Schoeller DA. Clinical characteristics influencing bioelectrical impedance analysis measurements. Am J Clin Nutr 1996:64:423S-7.

27 Sánchez-López AM, Noack-Segovia JP, Núñez-Negrillo AM, et al. Childhood obesity and its influence on sleep disorders: Kids-Play study. Int J Environ Res Public Health 2020;17:7948.

$28 \mathrm{Li}$ L, Zhang S, Huang Y, et al. Sleep duration and obesity in children: a systematic review and meta-analysis of prospective cohort studies. J Paediatr Child Health 2017;53:378-85.

29 Miller MA, Kruisbrink M, Wallace J, et al. Sleep duration and incidence of obesity in infants, children, and adolescents: a systematic review and meta-analysis of prospective studies. Sleep 2018:41 doi:10.1093/sleep/zsy018

$30 \mathrm{Seo}$ SH, Shim YS. Association of sleep duration with obesity and cardiometabolic risk factors in children and adolescents: a population-based study. Sci Rep 2019;9:9463.

31 Morrissey B, Taveras E, Allender S, et al. Sleep and obesity among children: a systematic review of multiple sleep dimensions. Pediatr Obes 2020;15:e12619.
32 Magee L, Hale L. Longitudinal associations between sleep duration and subsequent weight gain: a systematic review. Sleep Med Rev 2012;16:231-41.

33 Kaar JL, Schmiege SJ, Kalkwarf HJ, et al. Longitudinal assessment of sleep trajectories during early childhood and their association with obesity. Child Obes 2020;16:211-7.

34 Marceau K, Abel EA, Duncan RJ, et al. Longitudinal associations of sleep duration, morning and evening cortisol, and BMI during childhood. Obesity 2019;27:645-52.

35 Cappuccio FP, Taggart FM, Kandala N-B, et al. Meta-analysis of short sleep duration and obesity in children and adults. Sleep 2008;31:619-26.

36 Carrillo-Larco RM, Bernabé-Ortiz A, Miranda JJ. Short sleep duration and childhood obesity: cross-sectional analysis in Peru and patterns in four developing countries. PLoS One 2014;9:e112433.

37 Shi Z, Taylor AW, Gill TK, et al. Short sleep duration and obesity among Australian children. BMC Public Health 2010;10:609.

38 Eisenmann JC, Ekkekakis P, Holmes M. Sleep duration and overweight among Australian children and adolescents. Acta Paediatr 2006:95:956-63.

39 Grandner MA, Chakravorty S, Perlis ML, et al. Habitual sleep duration associated with self-reported and objectively determined cardiometabolic risk factors. Sleep Med 2014;15:42-50.

40 Wang F, Liu H, Wan Y, et al. Sleep duration and Overweight/Obesity in preschool-aged children: a prospective study of up to 48,922 children of the Jiaxing birth cohort. Sleep 2016;39:2013-9.

41 Moraleda-Cibrián M, O'Brien LM. Sleep duration and body mass index in children and adolescents with and without obstructive sleep apnea. Sleep Breath 2014;18:555-61.

42 Cespedes EM, Bhupathiraju SN, Li Y, et al. Long-term changes in sleep duration, energy balance and risk of type 2 diabetes. Diabetologia 2016;59:101-9.

43 Dashti HS, Scheer FA, Jacques PF, et al. Short sleep duration and dietary intake: epidemiologic evidence, mechanisms, and health implications. Adv Nutr 2015;6:648-59.

44 Poggiogalle E, Lubrano C, Gnessi L, et al. Reduced sleep duration affects body composition, dietary intake and quality of life in obese subjects. Eat Weight Disord 2016;21:501-5.

45 Mostazir M, Jeffery A, Hosking J, et al. Evidence for energy conservation during pubertal growth. A 10-year longitudinal study (EarlyBird 71). Int J Obes 2016;40:1619-26.

46 Collings PJ, Brage S, Ridgway CL, et al. Physical activity intensity, sedentary time, and body composition in preschoolers. Am J Clin Nutr 2013:97:1020-8.

47 Janz KF, Levy SM, Burns TL, et al. Fatness, physical activity, and television viewing in children during the adiposity rebound period: the lowa bone development study. Prev Med 2002;35:563-71.

48 Miller AL, Lumeng JC, LeBourgeois MK. Sleep patterns and obesity in childhood. Curr Opin Endocrinol Diabetes Obes 2015;22:41-7.

49 Hart CN, Cairns A, Jelalian E. Sleep and obesity in children and adolescents. Pediatr Clin North Am 2011;58:715-33.

50 Ding C, Lim LL, Xu L, et al. Sleep and obesity. J Obes Metab Syndr 2018;27:4-24.

51 Smith C, Galland B, Taylor R, et al. ActiGraph GT3X+ and actical wrist and hip worn accelerometers for sleep and wake indices in young children using an automated algorithm: validation with polysomnography. Front Psychiatry 2019;10:958.

52 Full KM, Kerr J, Grandner MA, et al. Validation of a physical activity accelerometer device worn on the hip and wrist against polysomnography. Sleep Health 2018;4:209-16. 